\title{
Overview on Chirality and Applications of Stereo-selective Dissolution testing in the Formulation and Development Work
}

\author{
Mukesh C. Gohel \\ Professor and Principal, L. M. College of Pharmacy, \\ P.O. Box 4011, Ahmedabad, 380 009, India
}

email correspondence to: mukeshgohel@hotmail.com

\begin{abstract}
The importance of chirality is considered in various areas such as purchasing of active pharmaceutical ingredient, selection of adjuvants in dosage form development, in vitro dissolution studies, stability studies, in vitro/ in vivo correlation (IVIVC), dossier preparation for technology transfer, ANDA/NDA applications and in scale up and post approval changes (SUPAC). The use of chiral selective dissolution testing is recommended for certain drugs that exist in racemic form in order assess the performance of a product, both in vitro and in vivo. An extension of the Biopharmaceutics Classification System $(B C S)$ is suggested for drugs that undergo chiral conversion after permeation through gastrointestinal wall, taking into account that the amount of active form of the drug reaching the receptor site is more important. It is suggested that the shelf life for chiral drugs shall be determined by considering the amount of degradation of the active form (R or $\mathrm{S}$ ) of the drug. This paper also gives a brief overview of work done on chirality from the viewpoint of formulation of dosage forms.
\end{abstract}

\section{Introduction}

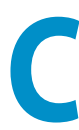

hiral molecules exist in a pair of stereoisomers (enantiomers). If a plane of polarized light is passed through a sample of each enantiomer, one will rotate the light to the left (levorotatory or (-)-enantiomer or R-form), and the other will rotate the light to the right (dextrorotatory or (+)-enantiomer or Sform). Rotation of light will not be observed if the light is passed through an equimolar mixture (racemic mixture). Scientists have started using prefixes such as lev- (e.g. levibuprofen), dex- (e.g. dexmethylphenidate), ar- (e.g. arbupivacaine) and es- (e.g. esomeprazole) to describe enantiomerically pure active pharmaceutical ingredients (API). A glossary of the selective terms related to stereochemistry is given in Table $1^{1}$.

Rentsch ${ }^{2}$ reported that about $56 \%$ of the synthetic drugs currently in use are chiral compounds, and $88 \%$ of these chiral synthetic drugs are used therapeutically as racemates. However, the recent trend in industry is to market the drugs in a pure enantiomeric form to give new life to old drugs by patenting the pure enantiomer.

Stephen ${ }^{3}$ reported that a worldwide sale of chiral drugs in single enantiomer was $\$ 133$ billion in 2000 . Forty percent of all dosage form drug sales in 2000 were of a single enantiomer. In 1999, the share was only one third. The advantages of marketing the drug in pure enantiomeric form are shown in Table $2^{4}$. Racemic switch or chiral switch means that racemic mixtures are redeveloped as single enantiomers ${ }^{3}$.

\section{Table 3: In Vivo Effect of Enantiomers}

Both enantiomers exhibit similar therapeutic properties (e.g. Promethazine, Flecainide) ${ }^{6}$

Only one isomer shows pharmacological activity (S-propranolol is a beta blocker) while the other one is inactive (R-propranolol) ${ }^{5}$

One type of isomer may show one type of pharmacological activity (S-penicillamine) and the other one shows toxicity (R-penicillamine) ${ }^{4}$ One type of isomer may show one type of pharmacological activity (R-methylphenylpropyl barbituric acid - anesthetic) and the other type shows convulsant effect ${ }^{5}$. 
Figure 1. Applications of Chirality in Formulation and Development

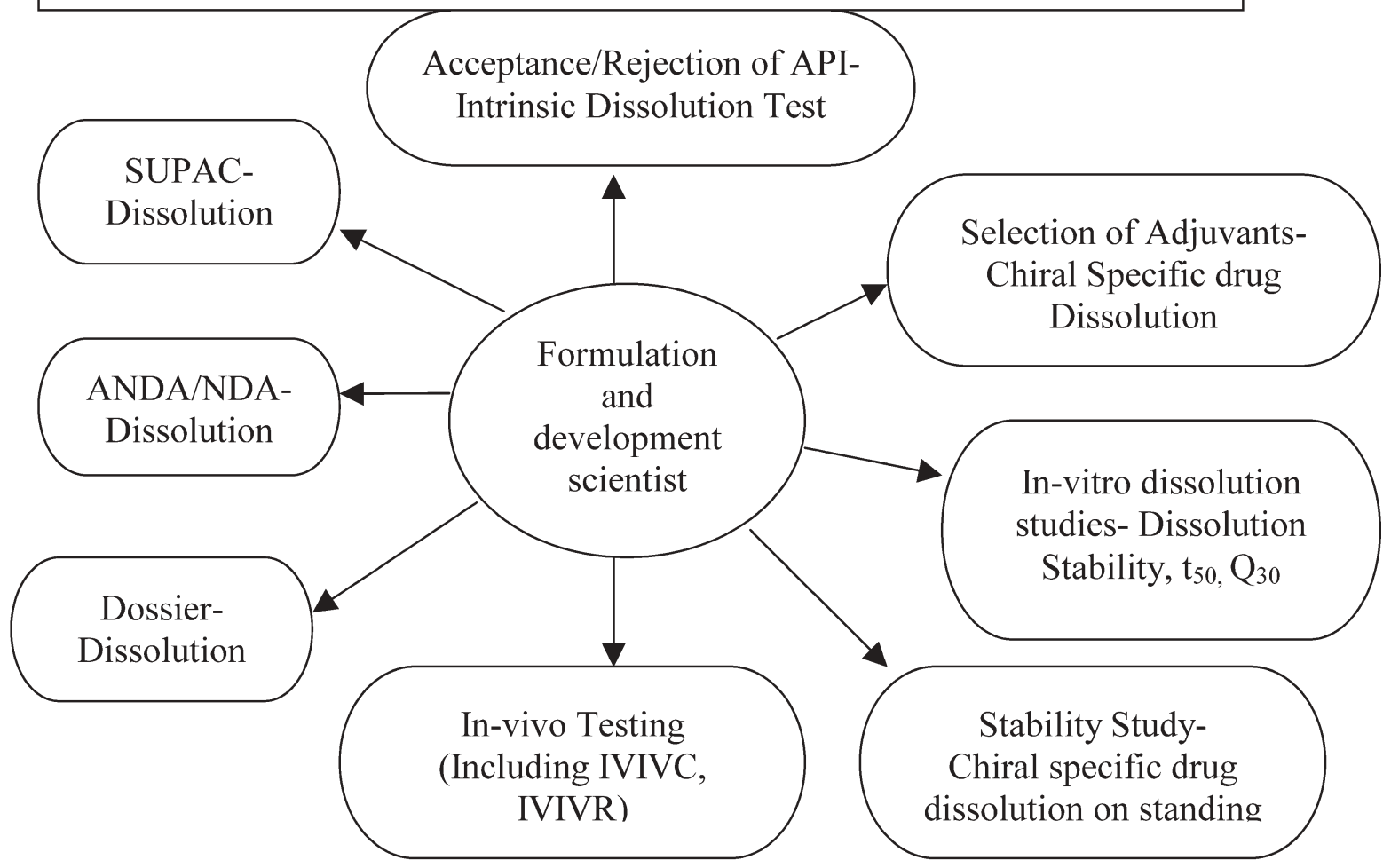

Enantiomeric forms of a drug can produce different therapeutic or adverse effects. Table 3 shows the different possibilities $^{4-6}$.

\section{Applications of Chirality from the viewpoint of Formulation Development:}

Many reports are found in literature on the applications of chirality in manufacturing of API and chiral separation. However, there are only few reports from the perspective of formulation and development (F\&D). Pharmaceutical companies around the world have registered significant success in racemic switching. Therefore, F\&D scientists now have to focus their attention on chirality. The applications of chirality from the viewpoint of F\&D are schematically shown in Figure 1.

\section{Acceptance/ Rejection of API:}

The analytical chemist generally carries out routine tests on drug purity (assay) and related impurities at the time of purchasing API. The chirally pure drugs should be quantitatively analyzed for the presence or absence of chiral impurities besides the routine determination of related impurities. Tao and Cooks ${ }^{7}$ recently reported that quantitative chiral analysis could be done by tandem MS. The method is rapid and requires very little sample. Lou $^{8}$ recently reported that it would be useful to determine enantiomers and other structurally similar drug impurities using one rather than two analytical methods.

The pharmaceutical companies should accept or reject a sample of API based on the ratio of the active/inactive enantiomers in the non-racemic mixtures. This ratio may change from one vendor to another. Most pharmaceutical companies do not consider this fact at the time of purchase. The success or failure of the formulation and development team may depend upon this ratio. The invitro dissolution test and in-vitro/in-vivo correlation (IVIVC) are the two major areas where the differences may be observed if an eye is not kept on chirality when API is purchased. The intrinsic dissolution rate of chirally pure API may prove to be a useful quality control parameter.

\section{Selection of Adjuvants:}

Formulation and development scientists add a number of adjuvants with diversified purposes in pharmaceutical formulations. Adjuvants are added to facilitate manufacturing, for functionality improvement, and to improve appearance and stability. The adjuvants may be either chiral or non-chiral in nature. The type and amount of the adjuvant may determine the functionality of the dosage form, especially the drug release rate. The characteristics of 


\section{Overview on Chirality and Applications ... continued}

adjuvant such as solubility, compressibility, surface area, porosity, etc. are considered today in F\&D. However, very little attention is given to the nature of adjuvant from the viewpoint of chirality.

A few references are cited below to show the effect of adjuvant type on drug release.

Solinis et $\mathrm{l}^{9}$. studied the release of salbutamol and ketoprofen enantiomers from hydroxypropylmethylcellulose (HPMC) K100M matrices containing two types of cellulose derivatives. The authors concluded that stereoselectivity is dependent on the amount of chiral excipient in the formulation.

Srichana and Suedee ${ }^{10}$ examined the in vitro dissolution of salbutamol from matrix tablets containing various chiral excipients such as gamma cyclodextrin, heptakis (2,6 di-Omethyl) -beta cyclodextrin, sulfobutyl-beta-cyclodextrin, HPMC and egg albumin. The formulation containing gamma cyclodextrin provided significant stereoselectivity throughout the dissolution profile. The release of eutomer R-salbutamol was higher than that of the distomer S-salbutamol from the gamma cyclodextrin tablets.

Suedee et al. ${ }^{11}$ monitored enantioselective in vitro release of propranolol. The influence of the method of polymer synthesis, drug to polymer ratio, $\mathrm{pH}$, and temperature on the release of two enantiomers was determined.

The study revealed that release of eutomer can be controlled via means of formulation. The distomer was retained in the dosage form.

Stereoselective interaction of ibuprofen was evaluated with chiral excipients such as HP-beta-cyclodextrin, tartaric acid, sucrose, HPMC, methylcellulose and a nonchiral excipient citric acid. The coprecipitates showed higher dissolution rate. The presence of chiral excipients did not cause stereoselective release of the drug ${ }^{12}$.

An area which can be tapped by the F\&D chemist is to control the drug release in the initial phase of drug dissolution by using a chiral adjuvant that exhibits binding affinity for the drug. This is sometimes necessary in real practice to match the in-vitro dissolution profile of the test product with that of the innovator's product. The objective may be achieved by preparing a bi-layer tablet. Applications of interaction between API and chiral excipient may be explored in the areas of sustained release buccal dosage form and colon drug delivery system. Teamwork between an expert analyst in chiral science and an F\&D scientist may open up many avenues in formulation development work.

\section{In Vitro Dissolution Studies and In Vivo Studies}

The industrial pharmacist considers dissolution testing an invaluable tool since it provides useful information throughout the formulation development work, i.e. from preparing the first trial formulation to the final marketed product. The dissolution profile of an innovator product and any limits prescribed by the health regulatory agencies are kept in mind while carrying out the developmental work.

Yonemochi et al. ${ }^{13}$ reported that the molecular arrangement in a crystal of enantiomers is different than that for racemic compound. If the difference in crystal structure results in a change in the melting point and heat of fusion, then the solubility of the pure enantiomers may be different from that of the racemic compound. The authors reported that the initial dissolution rate of racemic propranolol $\mathrm{HCl}$ was three times greater than that of enantiomers in distilled water.

For the drugs that exist in the racemic form where activity of the drug is attributed to one of the forms (R or S), we should not talk about the cumulative amount of drug $(R$ plus S) released from the dosage form but about the percentage of the active enantiomer released with respect to time. This is now very much possible with the advancements made in analytical techniques such as capillary electrophoresis, simulated moving bed chromatography, etc.

A single point dissolution specification such as $t_{50}$ (time required for $50 \%$ API dissolution) or $\mathrm{Q}_{30}$ (amount of API dissolved in $30 \mathrm{~min}$ ) is routinely employed as a quality control release test and it is also used for establishing dissolution stability on storage. For the drugs where only one of the forms (R or S) is active, the use of a stereoselective dissolution test is recommended for the calculation of $t_{50}$ or $Q_{30}$.

The results of chiral specific drug dissolution in a biorelevant dissolution medium or compendia media may be used for the selection of an appropriate formulation for in vivo studies or for convolution-deconvolution studies. Data may also be submitted to regulatory agency to obtain bio-waiver or for SUPAC for immediate release formulations.

After establishing the similarity of in vitro dissolution testing between a reference and a test product in different dissolution media, in vivo testing in man (bioequivalence testing) is ordered. It is a hard fact that many experimental formulations fail to establish an IVIVC. In such cases, reverse engineering is ordered. The major concern for any pharmaceutical company is the loss of time and money due to such failures. There may be multiple reasons for the failure of bioequivalence studies. In establishing Level A IVIVC for the products with significantly dissolution ratelimited absorption (and essentially complete absorption), one should consider the fraction of dose of active isomer dissolved versus the fraction of active isomer dose absorbed. The same approach may be used for calculation of parameters such as mean dissolution time (MDT) or mean residence time (MRT). We must also remember that the living body is a highly chiral environment. It is worthwhile to note here that optical isomers exhibit significant differences in their affinities for the receptor site, protein binding and biotransformation. 
Bui et al. ${ }^{14}$ assessed in vitro dissolution testing and bioequivalence of two formulations of chlorpheniramine tablets. Dissolution tests showed that both formulations were equivalent. A nonstereoselective, but not a stereoselective, approach indicated bioequivalence between the formulations.

A pure enantiomer may be converted to another form after absorption or it may remain in its original form. The $S$ - form of ibuprofen is the active form but significant $R$ to $S$ inversion takes place in the body. Therefore, even when ibuprofen is administered in racemic form, the drug exhibits $75 \%$ of the activity of the esibuprofen at the same dose level ${ }^{5}$. The use of stereoselective assay method is recommended in selected cases. The two enantiomers of thalidomide are inter-converted far too quickly ( $8 \mathrm{hr}$ ) in the liver ${ }^{5}$. S-thalidomide exhibits teratogenic effect whereas $R$ thalidomide is sedative. For thalidomide there is no point in giving pure enantiomer because of inter-conversion. It is worthwhile to note here that some drugs may not undergo in-vivo conversion ( $\mathrm{R}$ to $\mathrm{S}$ or $\mathrm{S}$ to $\mathrm{R}$ ). In such cases, enantioselective determination in bioequivalence testing is not the absolute requirement.

The biopharmaceutics classification system (BCS) guidance of US FDA classifies the drugs in four classes considering the solubility and permeability of drugs. Once the drug meant for oral use dissolves in gastro-intestinal fluid and subsequently permeates through the membrane, it enters into general circulation. One should remember that some drugs may undergo chiral conversion in blood. In such cases, the pharmacological action will depend upon the amount of unchanged active enantiomers reaching the receptors. Enantioselective analysis should be adopted in cases where there is the possibility of chiral conversion in blood. The third dimension can be added to $\mathrm{BCS}$, i.e. chiral conversion for the drugs where only one form (R or $S$ ) is active and the other form is inactive (Table 4). The drugs that fall under Class 1B will show superior action as compared to Class $1 \mathrm{~A}$. Each area shown in Table 3 may be explored in this way.

Polli ${ }^{15}$ reported that one intent of in vitro-in vivo relationship (IVIVR) is to learn about the relative contribution of dissolution to a product's overall absorption kinetics. Here also, chiral-specific dissolution can be used.

\section{Stability Study:}

In the FDA's policy statement for the development of new stereomeric drugs, it is mentioned that the stability protocol for enantiomeric drug substances and drug products should include a method or methods capable of assessing the stereochemical integrity of the drug substance and drug product ${ }^{16}$. However, once it has been demonstrated that stereochemical conversion does not occur, stereoselective tests might not be needed.

The stability study program must address the issue of

\section{Table : 4 Extension of Biopharmaceutics Drug Classification System}

\begin{tabular}{|c|l|l|l|l|}
\hline Class & Solubility & Permeability & \multicolumn{2}{|l|}{ Chiral Conversion* } \\
\hline \multirow{2}{*}{ I } & \multirow{2}{*}{ High } & \multirow{2}{*}{ High } & A & High \\
\cline { 4 - 5 } & & & B & Low \\
\hline \multirow{2}{*}{ III } & \multirow{2}{*}{ Low } & High & A & High \\
\cline { 4 - 5 } & \multirow{2}{*}{ High } & \multirow{2}{*}{ Low } & B & Low \\
\cline { 4 - 5 } & & & B & Low \\
\hline \multirow{2}{*}{ IV } & \multirow{2}{*}{ Low } & \multirow{2}{*}{ Low } & A & High \\
\cline { 4 - 5 } & & & B & Low \\
\hline
\end{tabular}

- *chiral conversion: An active form of drug is converted into an inactive form in bloodstream. For racemic drugs, the fraction of dose of active enantiomer reaching the receptor site is more relevant for pharmacological response.

- High:Higher amount of active enantiomer is converted to inactive form.

- Low: Lower amount of active enantiomer is converted to inactive form.

chiral inversion and racemization. Pomponio et al. carried out photostability studies on inclusion complexes of racemic nicardipine with beta-cyclodextrin and 2-hydroxypropyl beta-cyclodextrin. Beta-cyclodextrin showed photoprotective effect (UV-A and UV-B radiations); conversely 2-hydroxypropyl beta-cyclodextrin favoured the drug photodegradation. The photodegradation was found to follow apparent first-order kinetics and two different kinetic constants were obtained for the two different enantiomers ${ }^{17}$.

Interactions between enantiomers have also been described ${ }^{1}$. Racemization, or action or process of changing from an optically active compound or an optically inactive mixture of corresponding dextro (d-) and levo forms is a major factor in pharmaceutical stability ${ }^{18}$.

Shelf life of pharmaceutical formulation is fixed after considering $90 \%$ or $95 \%$ drug degradation under stated conditions of drug storage. This could be the amount of inactive/active enantiomer. For the racemic drugs where the action is attributed to one form (R or S), the scientist should talk about the amount of active enantiomers (R or S) degraded. We should not be surprised if the FDA starts assigning shelf life on the basis of degradation of active enantiomers since that seems to be more realistic.

Besides chemical stability, dissolution stability is also an equally important issue for popular dosage forms such as tablets and capsules. Many tablets show protracted disintegration on standing and such a change will generally result in change in dissolution rate. In select cases, the use of enantioselective dissolution is recommended to ascertain dissolution stability.

\section{Dossier for Technology Transfer}

The responsibility of the $F \& D$ team is not only to develop a functional formulation but also to prepare a well-docu- 


\section{Overview on Chirality and Applications ... continued}

mented dossier so that consistency from lot to lot is maintained in a marketed formulation. All the drugs that exist in racemic form and the drugs that are used in the pure enantiomer need special attention at the time of dossier preparation. The FDA and other applicable agencies' practices (ICH, Pharmacopoeia, etc.) shall be kept in mind while preparing the dossier. Good dossier preparation practice may prove to be useful at the time of inspection by the regulatory agencies.

\section{NDA/ANDA Application}

The current applicable guidance documents shall be kept in mind while preparing the applications. We should also keep in mind that increasing the desired activity should not be accompanied by a rise in the untoward effect. FDA requires toxicology testing on the racemate. The data of stereoselective dissolution testing should be submitted for the enantiomers that exhibit different action (e.g. methylphenylpropyl barbituric acid). There are endless opportunities and it is difficult to address all relevant issues in this communication. The current guidance documents should be consulted at the time of submitting an application.

\section{Summary}

Change in the formulation and development work may be reflected into change in drug dissolution and stability. The commonly used excipients such as beta-cyclodextrin, HPMC, maltodextrin, etc., may affect the drug release rate. The use of chiral selective dissolution testing or non-chiral dissolution testing should be considered on a case-by-case basis for chiral API. In the coming years, scientists will carry out research in arresting undesirable chiral conversion in the blood stream. The dose of API can be reduced if researchers are able to arrest conversion of an active form of a drug into an inactive form of a drug. Finally, it is concluded that chirality is going to be an important issue even in F\&D work, and the use of stereoselective dissolution testing is going to find widespread applications.

\section{Acknowledgement}

This paper is dedicated to my guide and mentor Dr. (Miss) Bhanooben M. Trivedi.

\section{References:}

1. Guidance for Industry:Stereochemical issues in Chiral Drug Development, Therapeutic Products Programme Guidance Document, Canada 2000, www.hcsc.ca/hpb-dgps/therapeuth .

2. Rentsch, K. M.,"The importance of stereoselective determination of drugs in the clinical laboratory." J. Biochem. Biophys. Methods, 54(1-3), 1-9, 2002.
3. Stephen, C. S.,"Chiral Pharmaceuticals.” Chemical and Engineering News, 79(40), 79-97, 2001.

4. http://www.imc-global.com

5. Houlton, S., "Chiral switches lead to blockbuster drugs.,"Manuf. Chemist, 73(1), 28-30, 2002.

6. Jamali, F., Mehvar, R., and Pasutto, F. M.,"Enantioselective aspects of drug action and disposition:therapeutic pitfalls."J. Pharm. Sci., 78(9), 693-713, 1989.

7. Tao, W. A. and Cooks, R. G., "Chiral analysis by MS." Analytical Chemistry, 75(1), 25A-31A, 2003.

8. Lou, W. J.,"Chiral Resolutions: Past, Present and Future.," The Ramanbhai Foundation First International Symposium in Recent Trends in Pharmaceutical Sciences, Symposium Companion, Ahmedabad, India, p.35, January 2003.

9. Solinis, M. A., De La Cruz, Y., Calvo, B., Hernandez, R. M., Cascon, A. R., Coni, I., Gurruchaga, M. D., and Pedraz, J.

L.,"Release of salbutamol sulphate and ketoprofen enantiomers from matrices containing HPMC and cellulose derivatives." Chirality, 14(10), 806-813, 2002.

10. Srichana, T. and Suedee, R.,"Evaluation of stereoselective dissolution of racemic salbutamol matrices prepared with commonly used excipients and 1H-NMR study." Drug Develop. Ind. Pharmacy, 27(5), 457-464, 2001.

11. Suedee, R., Srichana, T. and Martin, G.P. ,"Evaluation of matrices containing molecularly imprinted polymers in the enantioselective controlled delivery of beta blockers." J. Controlled Release, 66(2-3), 135-147, 2000.

12. Janjikhel, R.K. and Adeyeye, C. M.,"Dissolution of ibuprofen enantiomers from co-precipitates and suspensions containing chiral excipients." Pharm. Dev. Technology, 4(1), 9-17, 1999.

13. Yonemochi, E.,Yoshihashi, Y. and Terada, K.,"Quantitative relationship between stability, initial dissolution rate and heat of solution of chiral drugs." Pharm. Research, 17(1), 90-93, 2000.

14. Bui, T. H., Fernandez, C., Vu K., Nguyen, K. H. and Thuillier, A., Farinotti, R., Arnaud, P. and Gimenez, F.,"Stereospecific versus nonstereoselective assessment for the bioequivalence of two formulations of racemic chlorpheniramine.",Chirality, 12(8), 599-605, 2000.

15. Polli, J. E.,"IVIVR versus IVIVC," Dissolution Technologies, 7(3), 6-8, 2000.

16. http://www.fda/gov/guidances/stereo.html

17. Pomponio, R., Gotti, R., Bertucci, C. and Cavrini, V., "Evidences of cyclodextrin mediated enantioselective photodegradation of rac-nicrdipine by capillary electrophoresis." Electrophoresis, 22(15), 3243-3250, 2001.

18. Elizabeth B. V., Stability of Pharmaceutical products, Remington:The Science and Practice of Pharmacy, $20^{\text {th }}$ edition, 2000, Lippincott Williams and Wilkins, Maryland USA, p. 986. 